Original Article

\title{
Effect of Exercise Speed and Isokinetic Feedback on the Middle and Lower Serratus Anterior Muscles during Push-up Exercises
}

\author{
WON-GYU Yoo ${ }^{1)}$ \\ 1) Department of Physical Therapy, College of Biomedical Science and Engineering, Inje University: \\ 607 Obangdong, Gimhae, Gyeongsangnam-do 621-749, Republic of Korea
}

\begin{abstract}
Purpose] This study assessed the use of different exercise speeds and isokinetic feedback on the middle and lower serratus anterior muscles during push-up exercises. [Subjects] Ten male workers voluntarily consented to participate in the study. [Methods] The subjects performed push-up exercises under three conditions. Surface electrodes were placed on the dominant right side of the middle and lower serratus anterior muscles. [Results] The middle and lower SA muscle activities under condition 2 were significantly decreased when compared with those under conditions 1 and 3 . The middle SA activity under condition 3 was significantly increased when compared with that under condition 1. [Conclusion] This study suggests that proper selection of push-up exercise speed may be necessary for selective strengthening of the SA and that isokinetic feedback information obtained using an accelerator can help in selective strengthening of the middle SA.

Key words: Accelerator, Isokinetic feedback, Middle SA
\end{abstract}

(This article was submitted Oct. 7, 2013, and was accepted Nov. 25, 2013)

\section{INTRODUCTION}

Poor scapular stability and alignment are often associated with neuromusculoskeletal dysfunction in the neck and shoulder region. Scapulothoracic muscle weakness is a cause of scapular instability and can contribute to secondary subacromial impingement syndrome ${ }^{1,2)}$. Weakness of the serratus anterior causes poor scapular orientation and instability, contributing to pathological kinematics, such as impingement ${ }^{3,4}$ ). As a closed-kinetic chain exercise, the push-up requires no equipment and is effective for activating the proximal scapulothoracic musculature, as well as distal parts of the upper extremity ${ }^{4,5)}$. The push-up is considered an effective exercise to strengthen the upper extremities ${ }^{5)}$. Push-up exercise is used to induce scapular elevation, and upward rotation should have a minimal effect on the upper trapezius and selective stimulation of the serratus anterior ${ }^{4}$. Several studies have revealed the importance of scapular muscle strength, neuromuscular control, and scapular stability in normal shoulder function ${ }^{6}$. However, the best exercise speed for push-up exercises is uncertain. Thus, in this study, we assessed the use of different exercise speeds and isokinetic feedback on the middle and lower serratus anterior muscles during push-up exercises.

Corresponding author. Won-gyu Yoo (E-mail: won7y@inje. ac.kr)

C2014 The Society of Physical Therapy Science. Published by IPEC Inc. This is an open-access article distributed under the terms of the Creative Commons Attribution Non-Commercial No Derivatives (by-ncnd) License $<$ http://creativecommons.org/licenses/by-nc-nd/3.0/>.

\section{SUBJECTS AND METHODS}

The subjects were 10 males (age, 23.0 2.4 years (mean $\pm \mathrm{SD}$ ); height, $175.1 \pm 3.5 \mathrm{~cm}$; and body weight, $65.5 \pm 3.6 \mathrm{~kg}$ ) who consented to participate in this study. All subjects were right-side dominant, and none had any congenital deformity of the upper extremities or any orthopedic or neurological disorder. All subjects provided informed consent before participating. Informed consent was obtained from each subject. This study was approved by the Yonsei University Faculty of Health Sciences Human Ethics Committee. Muscle activities of the upper and lower serratus anterior muscles were recorded with an electromyography (EMG) system (Biopac Systems, Santa Barbara, CA, USA). All EMG signals were amplified and sampled at $1000 \mathrm{~Hz}$ using the AcqKnowledge software (ver. 3.9.1; Biopac Systems). The mean value of the EMG data for all tasks was expressed as a percentage relative to the maximal voluntary contraction. Surface electrodes were placed on the dominant right side of the middle serratus anterior (middle SA), at approximately the midaxillary line of the right side over the fifth rib, and the lower serratus anterior (lower SA), on the belly of the muscle that branched to the seventh rib. The subjects performed push-up exercises under three conditions. Condition 1 was a standard push-up exercise. Ten push-ups were performed, each taking $3 \mathrm{~s}$. We used the mean value of three randomly selected push-ups from the ten. Speed was controlled with a metronome at 60 beats/min, and the investigator initiated and stopped the EMG program at the start and end of the phase. Condition 2 was a speedy push-up exercise. Each push-up took $2 \mathrm{~s}$ to perform. The test procedure was otherwise the same as con- 
dition 1. Condition 3 was a push-up exercise with isokinetic feedback using an accelerometer. Each push-up took $3 \mathrm{~s}$ to perform. An isokinetic accelerometer feedback device was attached on the lateral right side of the trunk. This device provided information about isokinetic exercise speed. Another function provided a changing color (red or green) bar on a screen if the speed was too high or too low with regard to the speed threshold. Capacitive components of an accelerometer are commonly used to convert mechanical motion into an electrical signal. In the present study, the analog signal was converted to a digital signal with an A/D converter (NI USB-6009, National Instruments; Austin, TX, USA). The accelerometer detected linear motion and provided visual feedback through graphic software via a user-friendly interface using LabVIEW (National Instruments). We used the mean value of three successful performances of the isokinetic feedback exercise. The test procedure was otherwise the same as condition 1 . The order of exercise conditions was selected randomly. Participants were allowed $5 \mathrm{~min}$ of practice for each condition to familiarize themselves with performing push-ups at the appropriate velocity of movement. The SPSS software (ver. 12.0; SPSS, Chicago, IL, USA) was used for statistical analyses. Differences in the upper and lower SA under the three conditions were tested by repeated measures one-way ANOVA. The major effects were determined using Bonferroni's correction, and significance was set at $\mathrm{p}<0.05$.

\section{RESULTS}

The middle and lower SA muscle activities under condition 2 (middle, $28.3 \pm 13.2 \%$; lower, $24.5 \pm 12.9 \%$ ) were significantly decreased when compared with those under conditions (middle, $40.1 \pm 18.6 \%$; lower, $32.6 \pm 14.8 \%$ ) and 3 (middle, $45.9 \pm 18.6 \%$; lower, $34.7 \pm 15.1 \%)(\mathrm{p}<0.05)$. The middle SA activity under condition 3 was significantly increased when compared with that under condition 1 $(\mathrm{p}<0.05)$.

\section{DISCUSSION}

In this study, we investigated the effect of different exercise speeds and isokinetic feedback on the middle and lower serratus anterior muscles during push-up exercises. Our results showed that the middle and lower SA muscle activities under condition 2 were significantly decreased compared with those under conditions 1 and 3 . We consider that the speedy push-up exercise might not have activated the SA sufficiently. Finally, performing the push-up exercise quickly changed the amount of loading during it. The middle SA under condition 3 with isokinetic feedback showed the highest activation among the conditions. This study suggests that an isokinetic feedback device produced balance in the ascending and descending phases. A pushup exercise consists of an eccentric-descending phase and a concentric-ascending phase. According to a previous report, the descending phase showed significantly decreased middle SA and lower SA activities than the ascending phase $^{7)}$. During the descending phase of the push-up, these muscle components undergo eccentric contraction. Thus, the subject feels the descending phase as a comfortable phase. For this reason, most subjects performing push-ups under condition 1 performed a fast ascending phase during the 3-s standard push-up exercise when compared with the descending phase. According to a previous report, the descending phase (eccentric contraction) has been suggested to elicit fatigue in the targeted muscles ${ }^{8,9)}$. Some researchers have shown that the middle and lower parts of the serratus anterior muscle are important for stabilizing scapular movement, and that activating the middle and lower parts of the serratus anterior muscle may be an effective form of intervention $^{4,5,7)}$. Based on the result of this study, it can be suggested that the proper selection of push-up exercise speed may be necessary for selective strengthening of the SA and that isokinetic feedback information obtained using an accelerator can help in selective strengthening of the middle SA.

\section{ACKNOWLEDGEMENT}

This research was supported by Basic Science Research Program through the National Research Foundation of Korea (NRF) funded by the Ministry of Education, Science and Technology (No. 2012R1A1B4001058).

\section{REFERENCES}

1) Kibler WB: The role of the scapula in athletic shoulder function. Am J Sports Med, 1998, 26: 325-337. [Medline]

2) Schmitt L, Snyder-Mackler L: Role of scapular stabilizers in etiology and treatment of impingement syndrome. J Orthop Sports Phys Ther, 1999, 29: 31-38. [Medline] [CrossRef]

3) Mottram SL: Dynamic stability of the scapula. Man Ther, 1997, 2: 123131. [Medline] [CrossRef]

4) Ludewig PM, Hoff MS, Osowski EE, et al.: Relative balance of serratus anterior and upper trapezius muscle activity during push-up exercises. Am J Sports Med, 2004, 32: 484-493. [Medline] [CrossRef]

5) Lehman GJ, Gilas D, Patel U: An unstable support surface does not increase scapulothoracic stabilizing muscle activity during push up and push up plus exercises. Man Ther, 2008, 13: 500-506. [Medline] [CrossRef]

6) Kibler WB, McMullen J: Scapular dyskinesis and its relation to shoulder pain. J Am Acad Orthop Surg, 2003, 11: 142-151. [Medline]

7) Park SY, Yoo WG: Differential activation of parts of the serratus anterior muscle during push-up variations on stable and unstable bases of support. J Electromyogr Kinesiol, 2011, 21: 861-867. [Medline] [CrossRef]

8) Inman VT, Saunders JB, Abbott LC: Observations on the function of the shoulder joint. J Bone Joint Surg, 1944, 26: 1-31.

9) Beck TW, Kasishke PR 2nd, Stock MS, et al.: Neural contributions to concentric vs. eccentric exercise-induced strength loss. J Strength Cond Res, 2012, 26: 633-640. [Medline] 